\title{
La fatiga laboral en el ámbito de seguridad y salud laboral en el marco jurídico venezolano
}

\section{Labor fatigue in the field of safety and work health in the venezuelan legal framework}

\author{
Desireth Carolina Bracho-Paz \\ desicbracho@hotmail.com \\ Universidad Privada Rafael Belloso Chacín, Maracaibo \\ Venezuela \\ https://orcid.org/0000-0003-2630-4451 \\ Jennifer Liseth Quintero-Medina \\ jquintero@urbe.edu.ve \\ Universidad Privada Rafael Belloso Chacín, Maracaibo \\ Venezuela \\ https://orcid.org/0000-0003-0471-8418
}

Recibido: 15 de noviembre de 2019

Aprobado: 20 de diciembre de 2019

\section{RESUMEN}

La presente investigación tuvo como objetivo principal analizar la fatiga laboral en el ámbito de seguridad y salud en el trabajo en el régimen jurídico venezolano. Teóricamente el estudio estuvo sustentado en los enfoques de Rivas (2007), Servicio de Prevención de Riesgos Laborales y Medicina del Trabajo (2008), Cortes (2007), Serrano (2007), CRBV (1999), LOPCYMAT (2005), LOTTT (2012), entre otros. Metodológicamente, el tipo de investigación fue documental explicativo, teórica pura en base a la aplicación de la misma, con un diseño bibliográfico documental no experimental, siendo la población de carácter documental, al igual que técnica de recolección con revisión doctrinal y de leyes y análisis documental. Como conclusiones se obtuvieron que son diversos los síntomas que sirven de indicadores claros para determinar la presencia de la fatiga laboral en un trabajador o trabajadora, presentándose causas que pueden ser de tres tipos: causas por factores físicos, dentro de los cuales se presenta la iluminación y el ruido, por factores químicos y biológicos. La misma trae como consecuencias mayor ausentismo en trabajadores fatigados, mayor probabilidad de 
accidentes laborales en personas fatigadas, disminución del estado de alerta aun durante turnos diurnos, incremento del riesgo de enfermedades cardiovasculares y de otras índoles. $Y$ finalmente, en el marco legal venezolano que fomenta las buenas condiciones del ambiente laboral, representada por la CRBV (199), la actual Ley Orgánica de Prevención Condiciones y Medio Ambiente de Trabajo (2005) y la LOTTT (2012), no logra impulsar del todo la prevención para impedir que el riesgo de la ocurrencia de una enfermedad ocupacional.

Descriptores: Fatiga laboral; LOPCYMAT; enfermedad ocupacional.

\begin{abstract}
The main objective of this research was to analyze labor fatigue in the field of occupational safety and health in the Venezuelan legal system. Theoretically the study was based on the approaches of Rivas (2007), Service of Prevention of Occupational Risks and Work Medicine (2008), Cortes (2007), Serrano (2007), CRBV (1999), LOPCYMAT (2005), LOTTT 2012), among others. Methodologically, the type of research was documentary explanatory, pure theoretical based on the application of the same, with a documentary non-experimental bibliographic design, being the documentary population, as well as collection technique with doctrinal revision and laws and analysis documentary film. As conclusions were obtained that are diverse the symptoms that serve as clear indicators to determine the presence of the labor fatigue in a worker, presenting causes that can be of three types: causes by physical factors, within which the illumination is presented And noise, by chemical and biological factors. The same results in greater absenteeism in fatigued workers, greater probability of work accidents in fatigued people, decreased alertness even during daytime shifts, increased risk of cardiovascular and other diseases. Finally, in the Venezuelan legal framework that promotes good working environment conditions, represented by the CRBV (199), the current Organic Law on Prevention Conditions and Work Environment (2005) and LOTTT (2012), fails to promote Of all prevention to prevent the risk of the occurrence of an occupational disease.
\end{abstract}

Descriptors: Labor Fatigue; LOPCYMAT; occupational disease.

\title{
INTRODUCCIÓN
}

Las organizaciones empresariales, indistintamente de ser públicas o privadas, mundialmente han asumido como prioridad la seguridad y salud de los trabajadores, teniendo en consideración más que la productividad, el cumplir con los deberes 
establecidos de las diversas leyes que regulan la seguridad y salud de los empleados, al presentarse actualmente una época llena de incertidumbre, restricciones, problemas, amenazas y dificultades de toda índole, lo cual acentúa en el ser humano su afán de alcanzar mejor calidad de vida forzándose aumentar sus actividades laborales.

En Venezuela, con la entrada en vigencia de la Ley Orgánica de Prevención, Condiciones y Medio Ambiente de Trabajo, (LOPCYMAT, 2005), surge en la legislación laboral venezolana componentes de suma importancia dado que esta ley tiene como propósito establecer normas y lineamientos que permitan garantizar a los trabajadores y trabajadoras condiciones de seguridad, salud en su ambiente de trabajo adecuado, lo cual conlleva que el ejercicio pleno de sus facultades físicas y mentales mediante la promoción del trabajo seguro y saludable, así como la prevención de los accidentes de trabajo y enfermedades ocupacionales.

Siendo estos dos últimos, es decir, los accidentes y enfermedades ocupacionales definidos y consagrados en los artículos 69 y 70 de la LOPCYMAT, en el cual se determina que el primero de ellos, los accidentes, son todos sucesos que produzca en el trabajador (a), una lesión funcional o corporal permanente o temporal, inmediata o posterior, o la muerte resultante de una acción que puede ser determinada o sobrevenida en el curso del trabajo. De acuerdo con la Organización Mundial de la Salud, (2008), la salud ocupacional es una actividad multidisciplinaria dirigida a promover, proteger la salud de los trabajadores mediante la prevención, el control de enfermedades y accidentes, así la eliminación de los factores, condiciones que ponen en peligro la salud, de igual forma la seguridad en el trabajo. Además, procura generar, promover el trabajo seguro y sano, así como buenos ambientes en las organizaciones de trabajo realzando el bienestar físico mental, social de los trabajadores, respaldando el perfeccionamiento junto al mantenimiento de su capacidad de trabajo.

Otro aspecto vital es la relación que se desarrolla en el ambiente y salud en el trabajo es que en las empresas han contribuido aumentar los riesgos que estos procesos conllevan para la población en general y para el trabajador en particular, causando el deterioro de su salud, y dando lugar a la aparición de nuevos daños derivados del 
trabajo, tal es el caso de la fatiga laboral.

En lo que refiere a la fatiga laboral, esta es un factor que condiciona el desarrollo mental físico además del rendimiento y la productividad de las personas. Para García (2013) la fatiga es o conlleva directamente la disminución de la capacidad física y metal del trabajador, generalmente después de haber realizado un trabajo durante un tiempo determinado donde se pueden asociar varios factores como alta carga de trabajo, trabajo continuo, características de las tareas, capacidad mental del individuo, tiempo de respuesta, entre otros.

Particularmente en Venezuela, este fenómeno es considerado como una enfermedad ocupacional, y ello se encuentra establecido en la Norma Técnica para la Declaración de Enfermedad Ocupacional, aprobada Ministerio del Poder Popular para el Trabajo y Seguridad Social en el año 2008, y se encuentra clasificada en la misma como una afección por factores psicosociales, en donde intervienen diversos factores para darle su origen, especialmente los relacionados con el estrés.

Tomando en cuenta que la fatiga laboral es un factor que condiciona el desarrollo mental físico es primordial que la legislación laboral venezolano regule tan fundamental enfermedad dado que los trabajadores se ven expuestos a sobre carga en el trabajo, además, del incremento de las jornadas de trabajo, repercutiendo en la salud de los trabajadores, asimismo las condiciones del medio ambiente laboral en ocasiones son precarias observándose contaminantes físicos como son ruido, vibraciones, iluminación, presión atmosférica entre otras tantas variantes a lo que son expuestas los trabajadores. En razón a ello, la presente investigación tuvo como propósito fundamental analizar la fatiga laboral en el ámbito del régimen jurídico venezolano de salud y seguridad en el trabajo. En primer término, la salud y seguridad del trabajo a pesar de sus múltiples enfoques y tratamientos, se encuentra con una concepción más generalizada que los restantes basada en la concepción médica del término en sus tres aspectos: somático o fisiológico, psíquico y sanitario. 


\section{Fatiga laboral como riesgo psicosocial a la luz del ordenamiento jurídico}

Es algo común en estudios sobre riesgos ergonómicos y psicosociales, encontrar una referencia directa a la fatiga, presentada en sus distintas modalidades: física, mental, emocional, sensorial. La misma puede fungir como un desencadenante de dificultades, lesiones o enfermedades o también como consecuencia de la acción de ellas.

Comenta Rivas (2007, p. 303) la fatiga se identifica como la disminución de las funciones y capacidad de rendimiento de un órgano u organismo, determinado por el tipo, intensidad y duración de la carga o estrés que genera una solicitación, esfuerzo o gasto que necesita su recuperación o descanso. De acuerdo con el Servicio de Prevención de Riesgos Laborales y Medicina del Trabajo (2008), la fatiga laboral:

"es aquella que se origina en la relación persona - trabajo. La persona no puede separarse del trabajador (es la persona misma quien trabaja, se relaciona con sus amigos, disfruta y sufre, descansa, tiene un sueño reparador o se fatiga más aún) y determinadas actitudes, vivencias o experiencias fuera del ámbito estrictamente laboral van a condicionar tanto que se origine fatiga como la forma en que va a ser percibida".

De este modo habría al menos dos vías de intervención en la prevención de la fatiga: de un lado las técnicas y estrategias centradas en la persona, de otra las centradas en las condiciones de trabajo y su organización e incluso las que pusieran su acento en la interacción de una y otra. Todas estas circunstancias que giran en torno a la fatiga laboral, en cuanto a sus causas consecuencias y medidas de prevención, representan datos importantes para el estudio de la misma como una enfermedad de tipo ocupacional, reconocida por la Organización Panamericana de la Salud, dentro de las afecciones por factores psicosociales.

Este listado es el mismo publicado a nivel legal, en la Norma Técnica para la Declaración de Enfermedad Ocupacional, publicado y aprobado por el Ministerio de sobre el cual se rige el Ministerio del Poder Popular para el Trabajo y Seguridad Social, por ende, en Venezuela, la fatiga laboral es considerada como una enfermedad ocupacional por las 
autoridades competentes en el caso, bajo los estamentos de la Ley Orgánica de Prevención, Condiciones y Medio Ambiente de Trabajo (2005).

En base a lo expuesto, se puede considerar que la fatiga es un indicador de que algo debe ser atendido, bien en el trabajador, bien en las condiciones de trabajo o en su organización. Las consecuencias de no hacerlo pueden repercutir en la salud y bienestar; en la capacidad y eficiencia productivas; en los costes sociales y económicos.

\section{Síntomas de la fatiga laboral en materia de salud y seguridad laboral en Venezuela}

En la actualidad tanto la seguridad como la salud ocupacional son consideradas fundamentales en los entornos laborales dado que en estos momentos los empleadores de las organizaciones empresariales deberán proporcionar a sus trabajadores condiciones óptimas dentro de sus entornos laborales, donde estas dos categorías, es decir la seguridad como la salud, tienen como objetivo proporcionar a los trabajadores una mejor calidad de vida y óptimas condiciones de trabajo a fin de evitar que la salud de estos pueda resultar afectada por situaciones que ellos mismos crearon.

En lo que refiere a la salud, a pesar de sus múltiples enfoques y tratamientos el concepto más generalizado de salud, de acuerdo con Cortes (2007; p.63) viene dado por la opinión más generalizada que las restantes basada en la concepción médica del tratamiento en sus tres aspectos, somáticos o fisiológico, psíquico y sanitario. En lo concerniente a la salud ocupacional Serrano (2007; p.148) señala que "es una rama de la salud pública cuyo propósito es mantener el más alto grado de bienestar físico; mental y social de los trabajadores; cualquiera que sea su tipo de trabajo y su condición contractual." La misma, según Serrano (2007; p. 148) se traduce:

"En la acción como el conjunto de actividades preventivas, ejecutadas por un equipo multidisciplinario en el cual existe sinergia continua, utilizando estrategias, técnicas y herramientas médicas y no médicas, enmarcadas en el ordenamiento legal, dirigido hacia la promoción de la salud y seguridad, el sistema de identificación, detección, evaluación, prevención, control, mantenimiento y vigilancia de los factores de riesgo laboral, además de la defensa y crecimiento de la calidad de vida de los 


\section{CIENCIAMATRIA}

Revista Interdisciplinaria de Humanidades, Educación, Ciencia y Tecnología

Año VI. Vol. VI. N¹. Edición Especial. 2020

Hecho el depósito de ley: pp201602FA4721

ISSN-L: 2542-3029; ISSN: 2610-802X

Universidad Nacional Experimental Francisco de Miranda (UNEFM). Santa Ana de Coro. Venezuela

Desireth Carolina Bracho-Paz; Jennifer Liseth Quintero-Medina

\section{trabajadores."}

En razón a lo expuesto en líneas anteriores, se puede decir que cuando se habla tanto de salud laboral como de salud ocupacional, se está refiriendo al estado físico, mental y social, donde el trabajador puede resultar afectado por diferentes riesgos existentes en el ámbito laboral, produciéndose lo que se denomina hoy en día como fatiga laboral, que de acuerdo con Díaz (2009, p. 8) consiste en un agotamiento de la persona, tanto a nivel nervioso, psicológico, muscular, intelectual o sensorial, que tiene como causa más probable la continuidad de una tarea sin descanso de compensación adecuado, por lo que se producen como efectos la pérdida de la capacidad funcional, falta de resistencia, sensación de impotencia y de malestar.

Tomando en consideración, la definición expuesta por Ansberg (2000; p.123; citado por Quevedo, 2005), referente a la fatiga laboral se puede inferir que de la actividad laboral y las condiciones ambientales bajo las cuales el trabajador debe desarrollarlas pueden influir negativamente sobre su salud, ocasionando síntomas específicos producto de la exposición a condiciones que no se presenta en la misma magnitud en su vida social cotidiana.

Asimismo, Alonso (2010) menciona que para detectar la fatiga laboral se presentan síntomas y signos que la hacen manifestarse. Las molestias frecuentes son la falta de concentración, la irritabilidad, las sensaciones de mareo, los dolores de cabeza, la pesadez corporal, la falta de fuerzas físicas o algún trastorno digestivo. Igualmente puede reflejarse en forma de signos accesibles a la percepción de otros: las ideas confusas, los movimientos torpes, la acumulación de errores, el cambio de conducta o el descenso de los rendimientos.

De tal manera, los síntomas que se presentan en los empleados que sufren de la fatiga laboral como enfermedad ocupacional, presentan diversos síntomas que se reflejan no solo a nivel físico y sensorial, sino también en la forma de actuar dentro de su contexto laboral. No obstante, dentro de este trabajo se resaltan entre los síntomas algunos como insomnio, depresión, inapetencia, astenia, malestar físico, y somnolencia, que se asocian 


\section{CIENCIAMATRIA}

Revista Interdisciplinaria de Humanidades, Educación, Ciencia y Tecnología

Año VI. Vol. VI. N¹. Edición Especial. 2020

Hecho el depósito de ley: pp201602FA4721 ISSN-L: 2542-3029; ISSN: 2610-802X

Universidad Nacional Experimental Francisco de Miranda (UNEFM). Santa Ana de Coro. Venezuela

Desireth Carolina Bracho-Paz; Jennifer Liseth Quintero-Medina

directamente a la fatiga laboral.

Insomnio

El insomnio es la falta de sueño y es derivado por diversas causas, lo cual conlleva a que se produzca el mismo. De acuerdo con Sarrais y De Castro (2007, p. 296), definen al insomnio como un trastorno del sueño que consiste en la imposibilidad para iniciar o mantener el sueño, o de conseguir una duración y calidad de sueño adecuada para restaurar la energía y el estado de vigilia normal. El mismo, se encuentra asociado a una disminución del rendimiento laboral y un incremento de la tasa de accidentes de automóvil, y una mayor propensión a padecer enfermedades médicas.

\section{Depresión}

Estar deprimido es algo más que estar triste o que llorar mucho, o aún más severo que estar cansado. Aun cuando los tres tipo de situaciones están presentes en las manifestaciones que tiene un enfermos que padece de depresión. De acuerdo con Minsal (2006, p. 23), la depresión es:

Una alteración patología del estado de ánimo con descenso del humor que termina en tristeza, acompañada de diversos síntomas y signos de tipo vegetativo, emocionales, del pensamiento, del comportamiento y de los ritmos vitales que persisten por tiempo habitualmente prolongado. Con frecuencia tiende a manifestarse en el curso de la vida, con aparición de varios episodios, adquiriendo un curso fásico o recurrente con tendencia a la recuperación entre ellos.

\section{Inapetencia}

La inapetencia es entonces un grave problema que pone en peligro la salud del que la padece. De acuerdo con Haehl-Hering (2009, p. 45), la inapetencia o falta de ganas de comer, es un síntoma ordinario de las enfermedades del estómago. Los medios usados ordinariamente para despertar el apetito, suelen causar más bien el efecto contrario.

\section{Astenia}


La astenia, de acuerdo con Portela (2011), es un síntoma muy inespecífico, ya que puede aparecer en un sinnúmero de procesos, de base física y psicológica, e incluso presentarse también en la persona sana. De hecho, la astenia es uno de los síntomas más frecuentes también en personas sin patología severa de base, hasta el punto que llega a convertirse en el principal problema clínico para uno de cada diez pacientes de Atención Primaria.

\section{Malestar físico}

El estado de malestar a nivel físico, en el espacio laboral viene dado por una alta carga física, en donde las demandas de trabajo sobrepasan los límites de las capacidades del trabajador. De acuerdo con Enriques, Schneider y Rodríguez (2010; p. 432), el malestar físico consiste en la experiencia de sentirse mal, incluyendo las sensaciones de incomodidad y molestia. Las personas pueden sentir malestar físico y no tener una enfermedad identificable.

\section{Somnolencia}

La somnolencia implica una dificultad de mantenerse despierto, y por consiguiente de tener concentración en las actividades, tendiendo a manifestarse por el cierre involuntario de los ojos de la persona afectada, y el mismo es un problema que comúnmente se presenta en el área laboral. Para, Rosales y Castro (2010) la somnolencia es la tendencia de la persona a quedarse dormido, también conocido como la propensión a dormirse o la habilidad de transición de la vigilia al sueño. La presencia e intensidad de esta necesidad puede ser inferida por cuán rápidamente se inicia el sueño, cuán fácilmente es interrumpido y cuánto tiempo se duerme.

\section{Causas de la fatiga laboral en el entorno laboral}

La edad, aptitudes, fatiga, motivación, percepción, memoria, decisión y acciones entre otros, se consideran como factores humanos los cuales se estudian por medio de una serie de disciplinas con el objeto de analizar reacciones del cuerpo humano, así como las 
posturas y los movimientos durante el trabajo y el análisis del trabajo, para conocer procesos, cargas y su distribución del sistema.

En este sentido, Ramírez (2011; p. 283), el factor humano es la causa principal de la mayoría de los accidentes. La razón es el propio individuo, por desequilibrio psíquico o físico. Las primeras se originan en la personalidad del sujeto, cuya conducta y comportamiento están influidos por estímulos y motivaciones o por sentimientos antagónicos y negativos. $Y$ las segundas ocasionan un desgaste a nivel corporal cansancio, falta de energía y lentitud en las actividades. Es por ello, que los estímulos provienen de acusas externas, en tanto que la conducta es consecuencia de la propia integración del yo, lo que en circunstancias concretas llega a manifestarse en hábitos y actitudes fijas como consecuencia de la formación e influencia del entorno en que se desenvuelve el sujeto.

Cuando las condiciones físicas ambientales en la zona de trabajo no son adecuadas, su influencia sobre trabajador aumenta la accidentabilidad, aparte de las implicaciones técnicas y materiales que puede tener. Las condiciones de vida, la situación familiar y la salud propia o de los suyos, constituyen otros factores de riesgo.

\section{Medio Ambiente de Trabajo}

El trabajo es una actividad humana libre, sea física o intelectual, permanente o transitoria, que una persona natural ejecuta conscientemente al servicio de otra. Ramírez (2005; p.734), expresa que el medio ambiente de trabajo es el resultado de elementos como: el progreso tecnológico, la diversidad de equipos que producen ruidos, exhalaciones de gas, métodos modernos de organización de trabajo y disposición de plantas o distribución. Todo esto son factores que influyen en el medio de trabajo, cuyas características condicionan la actividad laboral del individuo en gran parte.

\section{Agentes físicos}

Las condiciones físicas ambientales actúan por medio de la interacción con las personas, de modo que hay que tener en cuenta que la participación de los factores individuales 


\section{CIENCIAMATRIA}

Revista Interdisciplinaria de Humanidades, Educación, Ciencia y Tecnología

Año VI. Vol. VI. N¹. Edición Especial. 2020

Hecho el depósito de ley: pp201602FA4721

ISSN-L: 2542-3029; ISSN: 2610-802X

Universidad Nacional Experimental Francisco de Miranda (UNEFM). Santa Ana de Coro. Venezuela

Desireth Carolina Bracho-Paz; Jennifer Liseth Quintero-Medina

como sociales y culturales, introducen variaciones relevantes en el efecto que pueden causar los agentes físico-ambientales. De acuerdo con Alcover, Martínez, Rodríguez y Domínguez (2004; p. 19),

Las condiciones del entorno físico o del ambiente han sido las que más atención han recibido tradicionalmente por parte de la Psicología del Trabajo y de las Organizaciones, a cuyo estudio también han contribuido la Psicología Ambiental y la Ergonomía. En ellas se incluyen tanto las variables relacionadas con el diseño de los lugares de trabajo, que obedecen en consecuencia a decisiones de los responsables organizacionales, como las variables derivadas de las características de la propia actividad laboral, y que son hasta cierto punto inevitables.

\section{Agentes químicos}

En la actualidad, la industria moderna requiere materias primas, todas de naturaleza química que en su manejo o transformación son capaces por sí mismas o mediante sus derivados, de desprender partículas sólidas, líquidas o gaseosas que absorbe el trabajador, produciendo el cuadro cronológico de la enfermedad profesional de que se trate. Para Hernández (2007, p. 763), el agente químico:

"es toda sustancia orgánica e inorgánica, natural o sintética que durante la fabricación, manejo, transporte, almacenamiento o uso, puede incorporarse al aire ambiente en forma de polvo, humo, gas o vapor, con efectos irritantes, corrosivos, asfixiantes o tóxicos y en cantidades que tengan probabilidades de lesionar la salud de las personas que entran en contacto con ellas".

\section{Agentes biológicos}

Para Hernández (2007; p. 487), los agentes biológicos tienen como origen la fijación dentro y/o fuera del organismo o la impregnación del mismo por animales protozoarios o metazoarios, parásitos o toxinas de bacterias que provocan el desarrollo de alguna enfermedad en el individuo, que proyectándose en el ambiente laboral se traduce en poco rendimiento del trabajador o la falta del mismo a sus funciones habituales. 


\section{Consecuencias de la fatiga laboral en los trabajadores}

Para Cortés (2007; p. 322), la carga de trabajo pude dar lugar a accidentes o fatiga laboral, tanto física o mental, manifestada esta última por los síntomas de irritabilidad, falta de energía y voluntad para trabajar, depresión, entre otros, acompañada frecuentemente de dolores de cabeza, mareos, insomnios, problemas digestivos.

De acuerdo con Carranza y Vallejo (2004; p. 93), las consecuencias que pueden materializarse en las siguientes situaciones: mayor ausentismo en trabajadores fatigados, mayor probabilidad de accidentes laborales en personas fatigadas, incremento del riesgo de enfermedades cardiovasculares, disminución del estado de alerta aun durante turnos diurnos, y de igual manera, la fatiga laboral no controlada se transforma en el síndrome de fatiga crónica de difícil manejo médico y de frecuentes recaídas.

\section{Accidentes}

Cortés (2007; p. 263), el accidente como la concreción o materialización de un riesgo, en un suceso imprevisto, que interrumpe o interfiere la continuidad de trabajo, que puede suponer un daño para las personas o a la propiedad. La causa inmediata de un accidente puede ser la falta de equipo de protección, pero la causa básica puede ser que el equipo de protección no se utilice porque resulta incómodo. Por otra parte, Fernández (2008; p. 490), expresa que el accidente laboral es todo suceso no querido ni deseado, que en un momento determinado irrumpe, de forma súbita e inesperada, el proceso productivo y produce daños a las personas.

\section{Incidentes}

Para Fernández (2008; p. 543), el incidente Es todo suceso no querido ni deseado, que en un momento determinado irrumpe de forma súbita e inesperada, el proceso productivo y es susceptible de producir daños o lesiones, pero las circunstancias concretas de la situación no dan tal resultado. Asimismo, Henao (2007; p. 83), expresa que los incidentes son sucesos deseados que disminuyen la calidad y la productividad en las empresas y 
deterioran la salud y la seguridad de las personas que laboran en éstas. Son considerados como errores en el sistema de administración de la empresa y evidencian la necesidad de resolver problemas en su funcionamiento.

\section{Enfermedades ocupacionales}

De acuerdo con Fernández (2008), una enfermedad ocupacional es el deterioro lento y paulatino de la salud del trabajador, resultado de una exposición prolongada al agente causante de la misma. Son de origen ocupacional o profesional cuando se puede establecer una relación causal entre el trabajo y la aparición de la enfermedad.

\section{Seguridad del trabajo}

Cortés (2007; p. 293) expresa que se entiende por seguridad del trabajo a la técnica no médica de prevención cuya finalidad se centra en la lucha contra los accidentes de trabajo, evitando y controlando sus consecuencias. Ese es precisamente su objetivo, luchar contra los accidentes de trabajo, y el mismo permite distinguir a la seguridad de otras técnicas no médicas de prevención, como la higiene y la ergonomía.

\section{Mecanismos de prevención de la fatiga laboral como enfermedad ocupacional}

Expresa Ramírez (2005, p. 241), que

Dado el accidente como una consecuencia de una situación funcional deficiente del sistema, se deben identificar las causas, para luego influir en ellas mediante medidas preventivas que permitan: prevenir las causas de perturbación de los elementos, mejorar la seguridad en el funcionamiento del sistema, y mejorar su interrelación. El punto de partida para la prevención de accidentes debe ser la creación y conservación del interés por la seguridad, en todos los niveles de la organización

Asimismo, Sánchez y otros (2007, p. 115), asevera que la prevención es el conjunto de actividades o medidas adoptadas en todas las fases de la actividad de la empresa con el fin de evitar o disminuir los riesgos derivados del trabajo. Un principio básico de la acción 
preventiva es combatir los riesgos en su origen, lo cual no siempre se consigue y debemos adoptar otras medidas. La protección de la seguridad y salud de los trabajadores en el trabajo pasa a ser objetivo principal, y ello va a exigir ir más allá del cumplimiento de deberes y obligaciones empresariales y, más aún, de la simple corrección de situaciones de riesgos que se manifiestan.

De igual manera, se pueden considerar algunas medidas preventivas en el trabajo, como lo son: procurar dotar a las tareas de un nivel de interés creciente, controlar la cantidad y la cantidad de la información tratada, adecuar, en relación con la tarea, el número y duración de los períodos de descanso, adecuada nutrición en relación con el consumo metabólico producido en el trabajo, acondicionar el sistema de trabajo de manera que los esfuerzos requeridos estén por debajo de los límites establecidos, elegir un mobiliario de trabajo adecuado a las tareas a desempeñar y que cumpla ciertos requisitos ergonómicos, y mantener dentro de los valores de confort los factores ambientales.

\section{Motivaciones humanas}

De acuerdo con Díaz (2008; p. 343), la motivación en el ser humano, desde las teorías psicoanalíticas, se relaciona con las pulsiones inconscientes que determinan la vida psíquica del individuo. Desde la fisiología es el resultado de una reacción homeostática que busca disminuir la tensión fisiológica que se genera ante un estado de insatisfacción o necesidad. En este sentido, la seguridad arranca como necesidad social de reparar los daños personales o lesiones causados por las condiciones de trabajo. La principal consecuencia que se deriva de los accidentes de trabajo y de las enfermedades profesionales la constituye la pérdida de salud del trabajador, lo que significa consecuencias indeseadas por el accidentado, su familia y la sociedad.

\section{Motivaciones económicas}

La buena marcha de una empresa se mide precisamente por el riguroso control que ésta ejerce sobre sus productos a partir del conocimiento de los gastos generales, precios de 
materias primas, costos de cada fase del proceso. Sin embargo, tal como lo acota Cortés (2007; p. 103), no es muy frecuente que dispongan de datos que les permitan conocer los costos de los accidentes y enfermedades profesionales acaecidas en la misma y de cuyo conocimiento se deducen una serie de consecuencias motivadoras de la seguridad.

Motivaciones legales

De acuerdo con Cortés (2007; p. 167), las motivaciones se basan en las consecuencias derivadas de la actuación legal que el Estado realiza a través de sus poderes legislativo, ejecutivo y judicial, para evitar y disminuir los daños derivados de los accidentes, estableciendo las responsabilidades y sanciones que se derivan de la actuación incorrecta en materia de prevención de riesgos laborales.

\section{Marco legal asociado a la seguridad y salud laboral en Venezuela}

Dentro de la legislación venezolana, se presentan una serie de regulaciones legales donde se basa la garantía de la salud y seguridad en el trabajo para los trabajadores, y bajo la cual se puede establecer un marco legal que regule la fatiga laboral como una enfermedad ocupacional presente en cualquier escenario laboral.

Este basamento legal se encuentra enmarcado dentro de la Constitución de la República Bolivariana de Venezuela (1999), que define en el Artículo 89, el precepto de que "EI trabajo es un hecho social y gozará de la protección especial del Estado. La ley dispondrá lo necesario para mejorar las condiciones materiales, morales e intelectuales de los trabajadores y trabajadoras ". En el caso de este reglamento legal, el mismo se encuentra representado por la Ley Orgánica del Trabajo, los Trabajadores y las Trabajadoras (2012).

\section{Contexto de la Ley Orgánica del Trabajo, los Trabajadores y las Trabajadoras (LOTTT, 2012) en el campo de Seguridad y Salud Laboral}

En primer lugar, la LOTTT (2012) explica en su artículo 1 que ella tiene como propósito proteger al trabajo como hecho social y garantizar los derechos de los trabajadores y de 
las trabajadoras como sujetos protagónicos de los procesos de educación y trabajo para alcanzar los fines del Estado democrático y social de derecho y de justicia, de conformidad con la Constitución Nacional.

Asimismo, en su artículo 3, menciona que esta Ley regirá las situaciones y relaciones laborales desarrolladas dentro del territorio nacional, de los trabajadores y trabajadoras con los patronos y patronas, derivadas del trabajo como hecho social, donde sus disposiciones en ningún caso, serán renunciables ni relajables por convenios particulares.

Por otra parte, la LOTTT (2012) establece como responsabilidad objetiva del patrono o patrona, en el artículo 43 que todo patrono o patrona garantizará a sus trabajadores 0 trabajadoras condiciones de seguridad, higiene y ambiente de trabajo adecuado, y son responsables por los accidentes laborales ocurridos y enfermedades ocupacionales acontecidas a los trabajadores, trabajadoras, aprendices, pasantes, becarios y becarias en la entidad de trabajo, o con motivo de causas relacionadas con el trabajo. La responsabilidad del patrono o patrona se establecerá exista o no culpa o negligencia de su parte o de los trabajadores, trabajadoras, aprendices, pasantes, becarios o becarias, y se procederá conforme a esta Ley en materia de salud y seguridad laboral.

Por otra parte, en la participación en salud y seguridad, en su artículo 44 que Los patronos o patronas están en la obligación de garantizar que los delegados y delegadas de prevención dispongan de facilidades para el cumplimiento de sus funciones, y que los comités de salud y seguridad laboral cuenten con la participación de todos y todas sus integrantes, y sus recomendaciones sean adoptadas en la entidad de trabajo En cuanto a las condiciones de trabajo, el artículo 156 establece que el trabajo se llevará a cabo en condiciones dignas y seguras, que permitan a los trabajadores y trabajadoras el desarrollo de sus potencialidades, capacidad creativa y pleno respeto a sus derechos humanos, garantizando: c) El tiempo para el descanso y la recreación. d) El ambiente saludable de trabajo. e) La protección a la vida, la salud y la seguridad laboral. f) La 
prevención y las condiciones necesarias para evitar toda forma de hostigamiento o acoso sexual y laboral.

Asimismo, la Ley establece Jornadas de trabajo inferiores por motivos de salud y seguridad en el trabajo, por lo que en su artículo 177 que el Ejecutivo Nacional podrá, en los reglamentos de esta Ley o por resolución especial, fijar una jornada menor para aquellos trabajos que requieran un esfuerzo excesivo o se realicen en condiciones de riesgo para la salud y seguridad de los trabajadores y trabajadoras.

\section{Regulaciones Ley Orgánica de Prevención, Condiciones y Medio Ambiente de Trabajo (LOPCYMAT, 2005)}

En la legislación nacional, existen ciertas disposiciones dentro de la Ley Orgánica de Prevención, Condiciones y Medio Ambiente de Trabajo (2005), la cual promueve la implementación del Régimen de Seguridad y Salud en el Trabajo, abarca la promoción de la salud de los trabajadores, la prevención de enfermedades profesionales y accidentes de trabajo, la atención, rehabilitación y reinserción de los trabajadores y establece las prestaciones dinerarias que correspondan por los daños que ocasionen enfermedades ocupacionales y accidentes de trabajo.

En este contexto la Ley Orgánica de Prevención, Condiciones y Medio Ambiente de Trabajo, expresa Castillo (2007; p. 295): "Que la misma se encuentra sostenida en algunos de sus articulados, en el que incluyen en forma implícita espacios extramuros ajenos al sitio de trabajo propiamente dicho, como por ejemplo lo expresado en el artículo 40 ordinal $1^{0}$ "Asegurar la protección de los trabajadores y trabajadoras contra toda condición que perjudique su salud producto de la actividad laboral y de las condiciones en que ésta se efectúa".

Debido a lo expuesto, los patronos se encuentran motivados a cumplir con las disposiciones legales del marco legislativo nacional, ya que es imperante la necesidad de dar orden a los flagelos que se presentan en la relación entre empleador y empleado, garantizando el derecho y beneficios de los trabajadores, incluyendo el 
desempeñar sus funciones en un ambiente cordial, de seguridad y con el mayor bienestar establecido por la ley.

Dentro de las distintas situaciones que puede afectar directamente a la salud de los trabajadores se presentan las enfermedades, por lo que en el Artículo 70 de la Lopcymat define lo que es una Enfermedad Ocupacional expresando que se entiende por la misma como los estados patológicos contraídos con ocasión del trabajo o exposición al medio en el que el trabajador se encuentra obligado a trabajar, tales como los imputables a la acción de factores psicosociales y emocionales (Entre otros), que se manifiesten por trastornos funcionales o desequilibrio mental (así como físico), temporales o permanentes.

Ahora bien, la Norma Técnica para la Declaración de Enfermedad Ocupacional emitida por el Inpsasel en el año 2008 expone 5 tipos de Afecciones por Factores Psicosociales: 1) Estrés ocupacional, 2) Fatiga laboral, 3) Agotamiento emocional (Síndrome), 4) Respuesta a Acoso laboral (Síndrome de Moobing) y 5) Trastornos no orgánicos del sueño.

Por lo cual, este listado es el mismo publicado a nivel legal, en la Norma Técnica para la Declaración de Enfermedad Ocupacional, publicado y aprobado por el Ministerio de sobre el cual se rige el Ministerio del Poder Popular para el Trabajo y Seguridad Social, en Venezuela, la fatiga laboral es considerada como una enfermedad ocupacional por las autoridades competentes en el caso, bajo los estamentos de la LOPCYMAT (2005) Los trabajadores y trabajadores que creen estar expuestos a este tipo de patologías por razones laborales deben como mínimo llevar sus planteamientos y denuncias (por escrito) ante los Delegados de Prevención, y estos últimos, hacer una análisis objetivos de las condiciones laborales a las cuales está expuesta el trabajador o trabajadora afectada, con el fin de verificar la veracidad de su situación, y posteriormente llevar dichos planteamientos ante el Comité de Seguridad para ser solucionadas. 


\section{METODOLOGÍA EMPLEADA}

Metodológicamente, el tipo de investigación fue documental Explicativa dado que, se orienta a recolectar información a partir de documentos escritos susceptibles de ser analizados, realizando evaluaciones precisas, por lo que la misma está orientada analizar la fatiga laboral en el ámbito de seguridad y salud en el trabajo en el régimen jurídico venezolano.

De igual manera, se presentó un diseño bibliográfico documental no experimental, cuyo propósito se estableció en relación con la consulta a los documentos legales publicados, basados en el desarrollo de contenidos asociados con la fatiga laboral como causante de la enfermedad ocupacional. Asimismo, se centra en el estudio de normativas, situaciones, instituciones e individuos dentro del contexto de la actividad laboral; donde no se manipulan a las condiciones ni categorías de manera deliberada.

La población para llevar a cabo la presente investigación, fue de carácter documental, por lo que se procedió a analizar en primer lugar examinar textos y luego el fundamento constitucional y legal de la influencia socio laboral de la fatiga laboral como enfermedad ocupacional en el marco jurídico venezolano.

Por otro lado, como técnica de recolección en el presente estudio se procedió a una revisión exhaustiva doctrinal de la fatiga laboral en el ámbito de seguridad y salud en el trabajo en el régimen jurídico venezolano, para obtener conocimiento conceptual de la fatiga laboral como causante de la enfermedad ocupacional. El análisis de los datos fue de tipo documental.

\section{CONSIDERACIONES FINALES}

Son diversos los síntomas que sirven de indicadores claros para determinar la presencia de la fatiga laboral en un trabajador o trabajadora, y los cuales pueden ser fenómenos que pueden percibirse a simple vista, materializados a nivel corporal, físico, así como psicosomático y sensitivo. Las sensaciones de falta de energía, astenia, insomnio, inapetencia somnolencia, son los síntomas comunes que se presentan en la masa 
trabajadora, y sobre los cuales se deben tener especial atención, los cuales son indicativos de una enfermedad ocupacional, como lo es la fatiga laboral.

Esto aún más, cuando legalmente, el organismo competente en cuanto a salud laboral a nivel nacional, considera dentro de sus normas y estatutos a la fatiga laboral como una enfermedad laboral, la cual debe ser tratada como tal y aplicar los procedimientos correspondientes por parte de las organizaciones para su prevención tratamiento y control, bajo el marco de la Ley Orgánica de Prevención Condiciones y Medio Ambiente en el Trabajo (LOPCYMAT, 2005), la cual representa la norma principal en cuanto a salud y seguridad laboral en el país.

Las causas de la fatiga laboral en el entorno laboral pueden ser de tres tipos: causas por factores físicos, dentro de los cuales se presenta la iluminación y el ruido, por factores químicos y biológicos, los cuales pueden presentarse de manera individual o converger unos con otros para crear un ambiente propicio para la aparición de la fatiga laboral en la masa trabajadora.

Asimismo, los factores de origen químicos, se encuentran presentes cuando se manipulan en los procesos productivos elementos químicos que entran en contacto directo con los empleados, y que en la mayoría de los casos consisten en materiales tóxicos que conllevan a la aparición de enfermedades o lesiones a nivel orgánico.

En cuanto a los factores biológicos, se concluye que son comunes encontrarlos en un ambiente donde se desenvuelven un grupo considerable de personas. Las enfermedades dentro de un ambiente de constante interacción, donde se realiza trabajo en equipo y se da un contacto interpersonal directa, las enfermedades, virus y bacterias son fácilmente transmisibles y contagiantes, por lo que el originar una enfermedad por esta causa es muy común y sencilla

Las posibles consecuencias de la fatiga laboral en los trabajadores dentro del entorno laboral pueden ser mayor ausentismo en trabajadores fatigados, mayor probabilidad de accidentes laborales en personas fatigadas, disminución del estado de alerta aun durante 
turnos diurnos, incremento del riesgo de enfermedades cardiovasculares y de otras índoles.

Por ello, la fatiga laboral, es una enfermedad que genera una serie de fenómenos a nivel orgánico, en donde los sistemas vitales se encuentran afectados, por medio de una reacción en cadena, debido a que todos trabajan como un todo organizado. Si el sistema cardiovascular se encuentra afectado, este puede influir en el funcionamiento del respiratorio, el digestivo y así sucesivamente, haciendo la aparición de una serie de síntomas y enfermedades a raíz de la fatiga.

En este contexto, se concluye que la salud laboral es el principal aspecto que se encuentra afectado por la fatiga laboral, representada como una enfermedad laboral, que a su vez, es la raíz que desencadena una serie de efectos negativos tangibles, de carácter operativo y material dentro del ciclo productivo de la empresa donde se desenvuelve un empleado que padece dicha enfermedad.

Representa entonces la fatiga laboral una disminución con respecto a la eficiencia en la realización de diferentes actividades y no solo laborales ya que como se ha expuesto, el trabajo tiene consecuencias diversas en la vida del hombre, ocasionando con ello una relación que converge entre esfuerzo y resultado, postergando la satisfacción del trabajador y extendiendo sus funciones laborales, en donde la persona, se convierte en un medio de producción que viabiliza la consecución de los diversos objetivos.

Los mecanismos de prevención de la fatiga laboral como enfermedad ocupacional en el mundo laboral, los problemas de fatiga mental se deben abordar desde el estudio de todas las condiciones que se presentan en el ambiente laboral del trabajo, de los requerimientos del mismo que recaen en el trabajador y de los recursos que tiene el individuo para poder cubrir con esas demandas. La prevención de la fatiga debe empezar desde el diseño de las condiciones de trabajo y la definición de los puestos de trabajo. En este contexto, se concluye que en el marco legal venezolano que fomenta las buenas condiciones del ambiente laboral, representada por la actual Ley Orgánica del Trabajo, los Trabajadores y las Trabajadoras (2012), La Constitución Nacional (1999) 
y la Ley Orgánica de Prevención Condiciones y Medio Ambiente de Trabajo (2005), no logra impulsar del todo la prevención para impedir que el riesgo de la ocurrencia de una enfermedad ocupacional como la fatiga laboral se presente, por lo que la norma establece las formas para diagnosticar, determinar las causas y certificar las consecuencias de la fatiga laboral, pero no ahonda sobre cómo prevenirla.

Las acciones presentes en la normativa legal para la prevención de la ocurrencia de una enfermedad ocupacional, resulta poco operativas, al ser medidas muy generales a aplicar, estas se reducen a evaluar y rectificar fallas y malas condiciones en el espacio laboral, evaluación de los factores de riesgo de accidentes y la planificación de la recreación, así como tiempo de descanso. Pero ello, resulta ser muy poco específico al momento de aplicarla directamente hacia la prevención de la fatiga laboral que, si bien es cierto ayuda, se necesita un grado más de especificidad para que la prevención sea efectiva.

Por ello, son inexactos los mecanismos de prevención de la fatiga laboral dentro del contexto legal nacional, a pesar de que la Ley Orgánica de Prevención, Condiciones y Medio Ambiente de Trabajo (2005) establece a los empleadores y empleadoras la responsabilidad de mantener buenas condiciones de trabajo para evitar la aparición de esta enfermedad psicosocial, pero no establece pasos o procedimientos exactos para garantizar la cultura preventiva o las acciones a tomar para asegurar la salud y seguridad laboral de sus empleados frente a la fatiga laboral.

En este sentido, las acciones encaminadas a la prevención de la fatiga desde el enfoque organizacional, se focalizan en el mejoramiento de las condiciones de trabajo y en la reformulación del contenido del puesto de trabajo. El mejoramiento de las condiciones de trabajo se apoyan en el estudio de las condiciones ambientales, de los elementos que forman parte del equipamiento o herramientas propias del puesto de trabajo, del tratamiento de la información y la distribución de los tiempos de trabajo, es decir las jornadas y horarios. 


\section{CIENCIAMATRIA}

Revista Interdisciplinaria de Humanidades, Educación, Ciencia y Tecnología

Año VI. Vol. VI. N 1 . Edición Especial. 2020

Hecho el depósito de ley: pp201602FA4721 ISSN-L: 2542-3029; ISSN: 2610-802X

Universidad Nacional Experimental Francisco de Miranda (UNEFM). Santa Ana de Coro. Venezuela

Desireth Carolina Bracho-Paz; Jennifer Liseth Quintero-Medina

\section{REFERENCIAS CONSULTADAS}

1. Alcover, Martínez, Rodríguez y Domínguez (2004). Introducción a la Psicología del Trabajo. Editorial McGraw Hill. Madrid, España.

2. Alonso, F. (2010). ¿Por qué trabajamos?: El trabajo entre el estrés y la felicidad. España. Editorial Díaz de Santos.

3. Carranza y Vallejo (2004). Fatiga Laboral. Revista Ergonomía Ocupacional S.L. Número 13. Monterrey, México.

4. Castillo, E. (2003) Medidas de Bioseguridad que practica el personal de Enfermería frente al riesgo de contraer Hepatitis B.92 Trabajo de grado para optar al título de Licenciado en Enfermería. Escuela Experimental de Enfermería Facultad de Medicina. UCV, Caracas.

5. Constitución de la República Bolivariana de Venezuela (1999)

6. Cortés, J. (2007). Técnicas de Prevención de Riesgos Laborales. Seguridad e higiene en el trabajo. Novena Edición. Editorial Tíbar, S.L. Madrid, España.

7. Díaz, M. (2008). Prevención de riesgos laborales: seguridad y salud laboral. España. Editorial Paraninfo.

8. Enríquez-Blanco, Schneider y Rodríguez (2010). Síndrome de Intestino Irritable y otros Trastornos Relacionados. Editorial Panamericana. México.

9. Fernández, R. (2008). Manual de prevención de riesgos laborales para no iniciados. Segunda edición. Editorial Club Universitario. Alicante, España.

10.García, V. (2013). Gestión de la prevención de riesgos laborales en pequeños negocios. COMT0112. España. IC Editorial.

11. Haehl-Hering (2009). Medicina Popular Homeopatica. Jain Publishers PVT. Ltd. Salamanca, España.

12. Hernández, F. (2007). Seguridad e Higiene Industrial. Editorial Limusa. Ciudad de México, México.

13. Ley Orgánica de Prevención, Condiciones y Medio Ambiente de Trabajo. Imprenta Nacional. Caracas, Venezuela (2005)

14. Ley Orgánica del Trabajo, los Trabajadores y las Trabajadoras (LOTTT, 2012). Gaceta Oficial 6076. Venezuela. 


\section{CIENCIAMATRIA}

Revista Interdisciplinaria de Humanidades, Educación, Ciencia y Tecnología

Año VI. Vol. VI. N 1 . Edición Especial. 2020

Hecho el depósito de ley: pp201602FA4721 ISSN-L: 2542-3029; ISSN: 2610-802X

Universidad Nacional Experimental Francisco de Miranda (UNEFM). Santa Ana de Coro. Venezuela

Desireth Carolina Bracho-Paz; Jennifer Liseth Quintero-Medina

15. Minsal (2006). Tratamiento de personas con depresión. Ministerio de Salud. Santiago, Chile

16. Norma Técnica para la Declaración de Enfermedad Ocupacional emitida por el Inpsasel en el año 2008

17.Portela, M. (2011). Astenia en cáncer avanzado y uso de psicoestimulantes. España. Anales del Sistema Sanitario de Navarra. Vol. 34.

18. Quevedo, A. (2005). Fatiga laboral y condiciones ambientales de una planta de envasado de una industria cervecera. Venezuela. Revista Salud de los trabajadores. Vol. 13.

19. Ramírez, C. (2005). Seguridad Industrial. Un enfoque integral. Segunda Edición Editorial Limusa. México.

20. Ramírez, C. (2007) Manual de Seguridad Industrial. México. Editorial Limusa.

21. Ramírez, C. (2011). Seguridad Industrial: Un Enfoque Integral. Tercera Edición. Editorial Limusa. México D.F.

22. Rivas, R. (2007). Ergonomía en el diseño y la producción industrial. Argentina. Editorial Nobuko.

23. Rosales, E. y Castro, J. (2007). Somnolencia y calidad del sueño en estudiantes de medicina de una Universidad peruana. Anuales de la Facultad de Medicina vol. 68. Universidad Nacional Mayor de San Marcos. Lima, Perú.

24. Sánchez y otros (2007). Seguridad en el Trabajo. Segunda Edición. FC Editorial. Madrid, España.

25. Sarrais y Castro (2007). El insomnio. Revista Anales. Vol. 30. Universidad de Pamplona. España.

26. Serrano, M (2007). Factores Psicosociales y Estrés en el Trabajo Hospitalario de Enfermería en Guadalajara. Revista Colombiana de Salud Ocupacional. Bogotá, Colombia.

27. Servicio de Prevención de Riesgos Laborales y Medicina del Trabajo (2008). Fatiga Laboral: Conceptos y Prevención. Delegación del Rector para Salud, Bienestar Social y Medioambiente. Universidad Complutense. Madrid, España. 


\section{REFERENCES CONSULTED}

1. Alcover, Martínez, Rodríguez and Domínguez (2004). Introduction to Labour Psychology. McGraw Hill Editorial. Madrid, Spain.

2. Alonso, F. (2010). Why we work: Work between stress and happiness. Spain. Editorial Díaz de Santos.

3. Carranza y Vallejo (2004). Work Fatigue. Revista Ergonomy Ocupacional S.L. Number 13. Monterrey, Mexico.

4. Castillo, E. (2003) Biosecurity measures practiced by Nursing personnel against the risk of contracting Hepatitis B.92 Undergraduate work for the title of Bachelor of Nursing. School of Nursing Faculty of Medicine. UCV, Caracas.

5. Constitution of the Bolivarian Republic of Venezuela (1999)

6. Cortes, J. (2007). Occupational Risk Prevention Techniques. Safety and hygiene at work. Ninth Edition. Editorial Tíbar, S.L. Madrid, Spain.

7. Diaz, M. (2008). Prevention of occupational hazards: occupational safety and health. Spain. Editorial Paraninfo.

8. Enríquez-Blanco, Schneider and Rodríguez (2010). Irritable Bowel Syndrome and other Related Disorders. Editorial Panamericana. México.

9. Fernandez, R. (2008). Workplace risk prevention manual for non-initiated ones. Second edition. Editorial University Club. Alicante, Spain

10. García, V. (2013). Management of the prevention of occupational risks in small businesses. COMT0112. Spain. IC Editorial.

11. Haehl-Hering (2009). Medicina Popular Homeopatica. Jain Publishers PVT. Ltd. Salamanca, España.

12. Hernandez, F. (2007). Industrial Safety and Hygiene. Editorial Limusa. Mexico City, Mexico.

13. Organic Law on Prevention, Conditions and the Working Environment. National Printing. Caracas, Venezuela (2005)

14. Organic Law on Labour, Workers and Workers (LOTTT, 2012). Official Gazette 


\section{CIENCIAMATRIA}

Revista Interdisciplinaria de Humanidades, Educación, Ciencia y Tecnología

Año VI. Vol. VI. N 1 . Edición Especial. 2020

Hecho el depósito de ley: pp201602FA4721 ISSN-L: 2542-3029; ISSN: 2610-802X

Universidad Nacional Experimental Francisco de Miranda (UNEFM). Santa Ana de Coro. Venezuela

Desireth Carolina Bracho-Paz; Jennifer Liseth Quintero-Medina

6076.

15. Venezuela.Minsal (2006). Treatment of people with depression. Ministry of Health. Santiago, Chile

16. Technical Standard for the Declaration of Occupational Disease issued by inpsasel in 2008

17. Portela, M. (2011). Asthenia in advanced cancer and use of psychostimulants. Spain. Anals of the Health System of Navarra. Vol. 34.

18. Quevedo, A. (2005). Fatiga laboral y condiciones ambientales de una planta de envasado de una industria cervecera. Venezuela. Revista Salud de los trabajadores. Vol. 13.

19. Ramirez, C. (2005). Industrial Safety. A holistic approach. Second Edition Editorial Limusa. Mexico

20. Ramirez, C. (2007) Industrial Safety Manual. Mexico. Editorial Limusa.

21. Ramirez, C. (2011). Industrial Safety: A Comprehensive Approach. Third Edition. Editorial Limusa. Mexico City.

22. Rivas, R. (2007). Ergonomics in industrial design and production. Argentina. Editorial Nobuko.

23. Rosales, E. and Castro, J. (2007). Sleepiness and sleep quality in medical students of a Peruvian University. Annual faculty of medicine vol. 68. Universidad Nacional Mayor de San Marcos. Lima, Peru.

24. Sanchez and others (2007). Security at Work. Second Edition. FC Editorial. Madrid, Spain.

25. Sarrais and Castro (2007). Insomnia. Anales Magazine. Vol. 30. University of Pamplona. Spain.

26. Serrano, M (2007). Psychosocial Factors and Stress at the Hospital Infirmary Work in Guadalajara. Colombian Journal of Occupational Health. Bogota, Colombia.

27. Occupational Risk Prevention and Occupational Medicine Service (2008). Work Fatigue: Concepts and Prevention. Delegation of the Rector for Health, Social Welfare and the Environment. Complutense University. Madrid, Spain. 
CIENCIAMATRIA

Revista Interdisciplinaria de Humanidades, Educación, Ciencia y Tecnología

Año VI. Vol. VI. N¹. Edición Especial. 2020

Hecho el depósito de ley: pp201602FA4721

ISSN-L: 2542-3029; ISSN: 2610-802X

Universidad Nacional Experimental Francisco de Miranda (UNEFM). Santa Ana de Coro. Venezuela

Desireth Carolina Bracho-Paz; Jennifer Liseth Quintero-Medina

2020 por los autores. Este artículo es de acceso abierto y distribuido según los términos y condiciones de la licencia Creative Commons Atribución-NoComercial-CompartirIgual 4.0 Internacional (CC BY-NC-SA 4.0)

(https://creativecommons.org/licenses/by-nc-sa/4.0/) 